\title{
Use of an online virtual environment in psychiatric problem-based learning
}

\author{
Jeremy Rampling, ${ }^{1}$ Aileen O'Brien, ${ }^{2}$ Keelyjo Hindhaugh, ${ }^{1}$ Luke Woodham, ${ }^{2}$ Sheetal Kavia ${ }^{2}$
}

The Psychiatrist (2012), 36, 391-396, doi: 10.1192/pb.bp.111.037630

${ }^{1}$ South West London \& St George's Mental Health NHS Trust;

${ }^{2}$ St George's, University of London

Correspondence to Jeremy Rampling (jeremy.rampling@swlstg-tr.nhs.uk)

First received 28 Oct 2011, final revision 14 Feb 2012, accepted 29 Mar 2012

\begin{abstract}
Aims and method To create a simulated patient with psychosis for psychiatric training within the online virtual environment of Second Life. After design and delivery of the scenario, medical students were asked to complete it and provide feedback.
\end{abstract}

Results A total of 24 students tried the scenario and gave feedback via an online survey. The project had been offered to 150 students so the take up was low. The feedback was predominantly negative with 53 critical responses to 32 positive ones. The consensus was that the scenario was cumbersome, did not imitate real life and was of little educational value. Multimedia representations of psychotic symptoms were more positively received and there may be scope for further development.

Clinical implications Interactive technology has a role in psychiatric education but we would not recommend the use of scenarios that rely predominantly on verbal communication within Second Life.

Declaration of interest None.
Virtual worlds are online simulated environments that provide the user with the opportunity to explore and interact with a potentially limitless range of objects and locations. Second Life (Linden Lab, www.secondlife.com) is one such world, it depicts a predominantly life-like visual environment with fantastical elements such as the ability of the subject to fly or teleport. Users in the virtual world, commonly known as residents, are represented by a personalised avatar and a range of interactions are possible. Avatars are able to interact with the environment and other objects, purchase goods and communicate using voice or text chat tools. The content and environments in Second Life are created by its residents and the platform provides tools for building objects and scripting interactivity that do not require significant technical expertise or specialist knowledge. The platform is free to access, allowing users to explore and visit existing locations in Second Life without any payment being required. However, users that wish to build their own objects and develop their own locations must rent land, for which there is a cost. As a result of it being free to access and the simplicity of its content creation tools, along with its support for a wide range of multimedia content, the platform has received significant interest from the education community who have used it to create a diverse range of interactive learning experiences. Coventry University was one of the first to use Second Life to provide healthcare education; ${ }^{1}$ since then many other institutions have followed. This includes the development of virtual scenarios and the organisation of mass gatherings, allowing for distance learning in the form of lectures or conferences available to a potentially global audience. Coventry University have created a virtual library, shop and theatre that have functional similarities to their real-life counterparts. Imperial College London have developed a virtual hospital within Second Life allowing medical students to assess patients, order investigations, discuss cases with colleagues and make diagnoses. ${ }^{2}$

Research into the use of Second Life in medical education is limited. However, Boston University have developed their site for provision of postgraduate medical education and published a small pilot study of a program for qualified doctors. ${ }^{3}$ Overall, the results were positive, with all participants agreeing that Second Life compared favourably to other online methods of delivering continued medical education. The main advantages identified included 'the added sense of presence afforded by a representative avatar, and the added real-life application provided by mockpatients. ${ }^{3}$

Problem-based learning (PBL) is increasingly used in undergraduate education. Virtual reality software can supplement PBL by creating an immersive environment where individual decision-making is afforded without reallife risk. ${ }^{4}$ Modern technology is able to develop hybrid learning methods to integrate PBL with online learning resources such as links to texts, videos or simulations.

St George's, University of London (SGUL) collaborated with Coventry University to explore the use of virtual worlds in the provision of problem-based education for paramedic and for health and social care students ${ }^{4}$ with generally positive feedback. Building from this, we aimed to develop a scenario in Second Life that would be beneficial to medical students on their psychiatry attachment. Previous 
feedback from SGUL students highlighted their anxieties in psychiatric history-taking, specifically in learning how to phrase sensitive questions and to ask about unfamiliar concepts. Many acutely psychotic presentations occur out of hours or in the community and students with little psychiatric experience are likely to feel uncomfortable with this presentation. Additionally, there is a professional challenge to interviewing patients without any background history that can provide intellectual satisfaction, potentially promoting an interest in psychiatry among students. In designing a virtual patient, our objective was to provide students with the opportunity to perform initial psychiatric assessment of a patient with first-onset, acute psychosis in the safety of a virtual environment. Our aim was to gather feedback from students about their perceptions of Second Life as an educational tool and to establish whether this generated or further stimulated an interest in psychiatry. We were also interested in receiving feedback about how to improve the tool.

\section{Method}

\section{Design}

The Centre for Enterprise and Innovation at SGUL funded $£ 14000$ for a 6-month project to develop a virtual psychiatry patient, to complete in February 2011. This sum funded the time of the staff to develop the tool and covered costs such as the annual rent of the land in Second Life. The design and construction of the scenario within Second Life were performed by two technical developers, while a consultant psychiatrist and two psychiatric registrars developed its content. Development of the scenario took an estimated $90 \mathrm{~h}$ and built upon the experience and systems developed as part of the PREVIEW project. ${ }^{5}$ The basis of the scenario is that the student is a junior doctor in psychiatry who has been asked to assess a young man whose family have raised concerns about his behaviour. He has stopped leaving the house and neighbours have heard shouting coming from his house. He has refused to come to the team base so the assessment is happening at home. It is made clear that in 'real life' the assessment would happen in pairs. The patient, on interview, describes delusions about climate change and reports hearing voices.

After this initial development, we invited a service user to offer creative suggestions and to comment on the authenticity and fairness of our representation of paranoid schizophrenia. The interview guide was shown to the service user who made recommendations about the patient's response based on his own experience of psychosis. He also gave suggestions about the décor and objects in the virtual flat.

A plot of land on the SGUL Island in Second Life was selected for the location of the scenario and a suitable home environment was created. The scenario was created using the open source PIVOTE (PREVIEW Immersive Virtual Training Environment) system (http://code.google.com/p/ pivote/), developed as part of the PREVIEW project ${ }^{5}$ to allow virtual scenarios to be designed and delivered into virtual worlds. In designing the virtual patient we were to create a non-playable character that appears visually to look like an avatar but, rather than being controlled by a user, responds to the comments and actions of other users. Users are able to interact with the non-playable character and the surrounding environment by clicking on key 'hot-spots' and in response will see text-based content and media, including images, sound and video, relating to the object clicked. They may also speak to the non-playable character using the text chat interface and receive a text-based response in the same way that they would if talking to a user-controlled avatar. This functionality is powered by a simple 'chatbot' engine that provides a response to user input using a predefined set of key words, phrases and triggers as criteria for determining an appropriate response. This approach imposed some practical limitations into the design of the case as we were unable to provide a purely open-ended interview, as the non-playable character could only respond in a finite number of programmed ways and then only to specific content within a user's questioning. The nonplayable character was also unable to determine a response or infer knowledge from previous statements, with each statement being processed in isolation. For example, referring to a previous topic by using a phrase such as 'How does that make you feel?' would have failed to elicit a relevant response as the non-playable character would have had no knowledge of what 'that' might be referring to. This makes lengthy exchanges or pre-scripted conversations with multiple questions impossible to deliver. Instead, these were launched as audio clips when students asked specific opening questions.

Accordingly, the scenario required careful design so that students were able to ask questions in the order of their choosing but that each scripted answer would make contextual sense in isolation, maintaining a sense of authenticity. The key phrases and triggers were selected based on anticipated and desirable questions, as well as previous experience of identifying potential problems or inconsistencies within the chatbot tool. Certain phrases and interview questions still provoked an inappropriate response from the non-playable character and to compensate for this, students were given a handout of suitable questions that could be used in this scenario, accessible via a link within the scenario itself. The handout suggested wordings of questions to ask. For example, 'Does it feel like people can control your thoughts?' would launch an audio clip of the patient's response and a subsequent conversation between the patient and an actor playing the visiting expert.

Environmental features were incorporated to harness the potential for interactivity of the scenario. The environment was designed to reflect the conditions and indicators that might be anticipated in a situation involving the patient's condition. For example, the patient's cupboards could be opened to reveal an absence of food. Similarly, clothes and newspapers could be found strewn around the floor and a general lack of cleanliness could be observed (see online Figs DS1 and DS2). Curtains were closed, although the scenario took place during daytime, suggestive of paranoia. Other features simulated the subjective experience of the patient via multimedia links. Selecting the newspaper, for example, would link to a visual representation of the patient's grandiose and persecutory delusions of reference 
about climate change. Interacting with the television or radio launched a video/audio sequence that further demonstrated the patient's perspective and the nature of his delusions, with references to himself appearing in news reports.

The scenario was not designed to be exhaustive and would not reflect a full psychiatric assessment. Instead, by exploring the patient's mood, thoughts, hallucinations, insight and risk, it was designed to guide students through more challenging aspects of the clinical interview.

Students were provided with custom written documentation and guides via the SGUL Virtual Learning Environment. These guides included details of how to navigate and interact in Second Life. The documentation also covered specifics about how to play scenarios and defined the interactions that students were able to perform in the environment. Specific details of the particular case were provided, including the location of the scenario, clinical background in the form of a referral letter and dialogue that the virtual patient was programmed to respond to. For those interested in viewing the program, the St George's Island is available at http://slurl.com/secondlife/St\%20Georges\%20 University/164/104/24 (this will require the user to register for Second Life).

\section{Testing}

Initial trials on a sample of three students were followed by testing on a cohort of fourth-year medical students on their psychiatric attachment, invited to complete the scenario in their own time. Three cohorts of 50 students were emailed giving them information about the project, as well as being informed of the project in lectures. A small number of the cohort (20 students) used the scenario in clinical PBL (CPBL) tutorial groups, working in groups of $6-10$. These sessions were facilitated by a higher specialist trainee in psychiatry who provided technical guidance on how to use the computer program without influencing the student's history-taking. This allowed the generation of learning objectives for discussion in the remainder of the CPBL session. After completion, students were invited to provide feedback via an anonymous online questionnaire that could be accessed from the scenario itself. The questionnaire was delivered using the web-based platform, SurveyMonkey, and consisted of 30 questions. These included multiple choice questions, Likert items and free-text responses.

\section{Results}

\section{Demographics}

A total of 24 students gave feedback via the anonymous online survey (11 female, 10 male, 3 gender not specified). The age range was 21-34 with a mean of 25. Thirteen were on the 5-year MBBS course, 9 were on the 4-year graduate entry course and 2 did not specify their course. Thirteen stated they had used Second Life before, whereas 8 had not, with 3 who did not answer this question.

Although 24 students responded, no question was answered by every student, so only between 21 and 23 responses were received for each question. Some responses were provided by students who had accessed the scenario in groups during CPBL sessions and therefore had not visited the scenario individually. An automated log of visitors to the scenario in Second Life was kept, which indicated that 23 unique visitors had seen the scenario. Table 1 details the participants' attitudes towards the training and guidance material provided and Table 2 their attitudes towards the clinical and academic usefulness of the scenario.

\section{Open feedback}

The responders were invited to comment in free text giving up to three advantages and three disadvantages of the exercise (Table 3). They were also asked to consider whether they felt the scenario could be used differently and ways they felt it could be improved.

The disadvantages outweighed the advantages with 53 critical responses to 32 positive ones from 20 and 18 participants respectively. One of the most commonly cited disadvantages was that the procedure was time consuming, both in setting up an avatar and learning to navigate through Second Life. Alongside this, many reported the program itself to be too slow. Other themes relating to the program were that the user interface was hard to navigate and that the program did not allow for flexibility. This led to complaints about the nature of the communication function with the virtual patient, which was not deemed to be an accurate simulation of a clinical interview.

Table 1 Scaled attitudes towards the training and guidance material provided to complete the scenario

\begin{tabular}{|c|c|c|c|c|c|c|}
\hline Training and guidance & $\begin{array}{l}\text { Strongly } \\
\text { agree }\end{array}$ & Agree & Neutral & Disagree & $\begin{array}{l}\text { Strongly } \\
\text { disagree }\end{array}$ & Total \\
\hline $\begin{array}{l}\text { 1. The training provided me with enough information to } \\
\text { introduce me to Second Life }\end{array}$ & 0 & 5 & 8 & 4 & 4 & 21 \\
\hline $\begin{array}{l}\text { 2. I can navigate my way in the Second Life environment } \\
\text { confidently }\end{array}$ & 0 & 5 & 2 & 11 & 4 & 22 \\
\hline $\begin{array}{l}\text { 3. I can communicate effectively in the Second Life } \\
\text { environment }\end{array}$ & 0 & 6 & 3 & 6 & 7 & 22 \\
\hline $\begin{array}{l}\text { 4. All the necessary components to interact with the Second } \\
\text { Life scenarios were introduced through the guide }\end{array}$ & 0 & 3 & 12 & 3 & 4 & 22 \\
\hline $\begin{array}{l}\text { 5. It is important that the Second Life scenario is facilitated by } \\
\text { a tutor }\end{array}$ & 5 & 9 & 3 & 6 & 0 & 23 \\
\hline $\begin{array}{l}\text { 6. An advantage of the Second Life scenario is that I can } \\
\text { access it at my own convenience }\end{array}$ & 2 & 12 & 3 & 3 & 3 & 23 \\
\hline
\end{tabular}


Table 2 Scaled attitudes towards the clinical and academic usefulness of the scenario

\begin{tabular}{|c|c|c|c|c|c|c|}
\hline Usefulness & $\begin{array}{c}\text { Strongly } \\
\text { agree }\end{array}$ & Agree & Neutral & Disagree & $\begin{array}{l}\text { Strongly } \\
\text { disagree }\end{array}$ & Total \\
\hline \multicolumn{7}{|l|}{ Clinical } \\
\hline \multicolumn{7}{|l|}{ 1. The exercise helped familiarise me with taking } \\
\hline \multicolumn{7}{|l|}{ 2. The exercise helped me structure the questioning in the } \\
\hline psychiatric history & 0 & 1 & 5 & 10 & 7 & 23 \\
\hline 3. I feel more confident taking a psychiatric history now & 0 & 0 & 6 & 12 & 5 & 23 \\
\hline \multicolumn{7}{|l|}{ 4. The Second Life scenario is a relevant resource in } \\
\hline \multicolumn{7}{|l|}{ 5. The exercise accurately reflects taking a psychiatric } \\
\hline history & 0 & 1 & 8 & 7 & 7 & 23 \\
\hline \multicolumn{7}{|l|}{ Knowledge base } \\
\hline 1. The exercise improved my knowledge of schizophrenia & 0 & 5 & 4 & 11 & 3 & 23 \\
\hline 2. The exercise increased my interest in psychiatry & 0 & 0 & 7 & 12 & 4 & 23 \\
\hline 3. Second Life is valuable for my revision for my exams & 0 & 2 & 3 & 9 & 9 & 23 \\
\hline \\
\hline \multicolumn{7}{|l|}{ 5. Overall, Second Life's effect on my learning process was } \\
\hline effective & 0 & 1 & 3 & 10 & 9 & 23 \\
\hline
\end{tabular}

Students liked the fact that the scenario could be accessed at any time and in their own home and commented positively on its interactivity. The most well-received educational elements of the exercise were the visual representations of psychotic symptoms such as delusions of reference with five students identifying this as an advantage and one reporting that it encouraged discussion of symptoms. Two felt that it may be a useful overview of a psychiatric assessment to be given either to preclinical students or at the start of their clinical attachment.

Students were asked to identify scope for improvement with only seven replies. Three commented on the flawed mode of communication, requesting freedom to interview in a more realistic and conversational manner. One suggested that the exercise would work better as a simple text program with a decision-tree rather than the text-box means of interacting with the avatar. Two stated that increasing the speed of the program was their most pressing concern.

Fourteen students gave feedback when asked to comment on suitable uses for the scenario. Two suggested that it may be useful in the first session of an interactive CPBL session with a third also suggesting its use in a tutorled group. Two felt it may be useful in training preclinical students regarding clinical skills and one felt it may be used in revision. Five respondents commented that they did not believe that there was a place for this kind of exercise in their medical education.

\section{Discussion}

The project aimed to identify whether there is a role for virtual worlds in the education of medical students in the field of mental health to complement more traditional methods such as PBL. Such an approach has proven successful within other aspects of healthcare education ${ }^{1,4,6}$ but this is the first time we are aware of where it has been specifically adapted to psychiatry. The novelty of this project sparked much interest among peers in psychiatric practice, but this enthusiasm did not transfer to the students who served as participants.
As this was intended to be a non-exhaustive introduction to psychiatric history-taking, we were predominantly interested in feedback from students about their perceptions of it as a useful educational tool and whether they felt it would stimulate interest in the subject of psychiatry. It is important to stress that it was never intended to replace 'hands-on' clinical teaching.

Table 3 Categorised open response

\begin{tabular}{lc} 
Response & $n$ \\
\hline Advantages & 32 \\
Convenient/can be done in own time/can be & 12 \\
accessed anywhere & \\
Good illustrations of ideas of reference/ & 5 \\
describes patient's perspective & 3 \\
Interactive & 2 \\
Interesting scenario & \\
Good overview if done at start/good for & 2 \\
students not yet on the wards & 1 \\
Discussion of psychiatric symptoms encouraged & 1 \\
Makes me think about what to do next & 1 \\
Aesthetically pleasing & 1 \\
Novel & 1 \\
Quick & 1 \\
Audiovisual research & 1 \\
Fun to fly about & 1 \\
\hline Can replay & 53 \\
\hline Disadvantages & 7 \\
Time consuming & 7 \\
Not user friendly & 7 \\
Plow & 7 \\
Inflexible/rigid/cannot go off topic & 6 \\
Unrealistic & 5 \\
Hassle/frustrating & 2 \\
Wvercomplicated & 2 \\
Clunky/cumbersome & 2 \\
Loor structure and layout & 1 \\
Unrealistic movements & 1 \\
Difficult to set up & 1 \\
No face-to-face contact at all effective & 1 \\
\hline
\end{tabular}


The feedback was predominantly negative, with a general sense that the program was difficult to use and that it offered little clinical or educational advantage. Responses to set statements show that the students did not find the experience interesting or thought provoking, although some of their free-text response was more positive and may help to identify what did work well about this project. As has been previously identified with virtual worlds such as Second Life, ${ }^{6}$ the high technology demands were a substantial barrier to effective use, with students complaining that the program was slow.

\section{Technical limitations}

There were many complaints that the program was cumbersome; it took students a long time to register for the site and to learn how to navigate the environment and, even after the online tutorial and training, they did not generally feel confident navigating and communicating within Second Life (Box 1). The speed of the program was also mentioned as a negative point, which might reflect the speed of broadband available or the processor capabilities being used by our students. The difficulty in learning how to use Second Life may represent a flaw with the program, but it may also identify flaws provided by our training or specific confounders of our student group. Previous work with a class of paramedic trainees demonstrated that they were more confident with the program and felt that the skills needed to use it could be learnt in a hour. ${ }^{6}$ The participant group in that study had learnt how to use Second Life in the presence of a tutor and it may be that facilitation is an important factor in satisfaction and confidence in this exercise. Toro-Troconis et al recommended that the students have at least $4 \mathrm{~h}$ of exposure to Second Life before using educational tools in this environment. ${ }^{7}$

The communication interface was not well received. In its current form, communication with a non-playable character is unable to provide a real-life simulation of history-taking because of the necessity of providing finite responses to specific questions. In psychiatry this is particularly problematic as the psychiatric history does not follow a linear path in the manner that, for example, a paramedic assessment might. As such, students are unable to structure the interview as they would in reality and are unable to ask questions in their own words, limiting the sense that they are making their own decisions. The situation, therefore, becomes less immersive and more akin to observation than practice, thus negating some of the perceived benefits of the virtual world design. Increasing the range of potential responses by populating the scenario with more content, even within the limitations of the existing chatbot engine, would diminish the number of times students received irrelevant responses to their questions. However, it would not resolve those issues relating to the lack of persistence in the conversation, i.e. the system's inability to cope with presumed knowledge implied by the conversational context, including phrases such as 'that' to describe a previously explicitly named subject. This could be addressed by using more advanced chatbot and artificial intelligence systems, although these are not currently available in the PIVOTE system.

\section{Box 1 Feedback on technical aspects}

'I couldn't follow the instructions. It appeared I was using a different version of Second Life. I enlisted the help of a techy friend and still we found it impossible to enable all the features.'

'It took ages to set up and then I couldn't make it work. All rather frustrating ... Too stilted to be worth the investment of time ... the room and interactive notes are great though so it's a shame.'

'I think the SL [Second Life] implementation is clunky and the whole SL environment reduces accessibility for students.'

\section{'Slow performance on older computers.'}

'Can't have a natural conversation.'

'Good concept but you can't allow the conversation to flow in your way, you have to make sure you use the key words.'

'Does not mimic real life.'

\section{Worth as an educational tool}

Students were resounding in their dismissal of Second Life as a tool for medical education. It was seen as a poor imitation of psychiatric clerking and of little clinical relevance. Yet a minority of students felt that it had increased their knowledge of schizophrenia and there was positive feedback regarding the interactivity and particularly the visual representations of psychotic symptoms (Box 2). Some students saw a use for the tool as an introduction to the topic and that it could be used with preclinical students or in PBL early in their psychiatric attachment, possibly before contact with a real-life patient. Anecdotally, the CPBL tutor reported that the scenario proved very useful for prompting discussion of psychotic symptoms and generating learning objectives within the session.

The visual representation of psychotic symptoms was the most positively received aspect of this program. We had designed an interactive newspaper, television and radio to depict first-person experience of ideas of reference. This role of the program could be developed as a separate educational resource designed to teach phenomenology to medical students and psychiatry trainees, as well as harness further interest in the topic. It might then find its place as a complementary resource to the myriad of other learning aids available in medical education. An immersive virtual world may not be necessary for such developments, and there is scope to design more multimedia representations of psychiatric symptoms to be viewed online at the students' convenience.

Although no student answered that the scenario had increased their interest in psychiatry, when offered the chance to respond in free-text some reported that they found the scenario itself interesting and one described it as 'fun'. This perhaps suggests that, if the frustrations with the program could be reduced, it may have a role in developing interest in the specialty. This could potentially be expanded to encourage recruitment to psychiatry at the level of A-level students.

Other studies have included a pre- and post-evaluation questionnaire $^{4}$ and this may have been useful here to establish whether any views about the psychiatric history changed after trying the Second Life tool. 


\section{Scope for improvement or adaptation}

There are aspects of Second Life that cannot be altered. For example, the navigation and communication functions are fixed by the program. If the time taken to learn the controls and the limitations of communicating with a non-playable character are deemed unacceptable, then an alternative program may have to be identified if such a project is to be further developed. This would still allow for some of the interactive elements such as multimedia representations of psychiatric symptoms but is likely to reduce the planned immersion in a virtual world and make a subtle change to the nature of the decision-making by prompting with select and finite options.

If Second Life were to be used, students were overwhelmingly in favour of it being done so in a group and the majority believed that it needed to be facilitated by a tutor (Box 2). This is consistent with previous work with paramedic students, ${ }^{5}$ although it would detract from one of the main perceived positives - that it can be completed anywhere and anytime. Those who could foresee a role for Second Life in their education looked upon it as a tutor-led tool to be given very early in their education in psychiatry, so if we were to design it further it should be targeted at students with little or no clinical experience in the subject.

\author{
Box 2 Some positive and negative feedback \\ Positive feedback \\ 'Encouraged discussion of illusions, hallucinations, delusions.' \\ 'Good illustration of ideas of reference.' \\ 'Visual representation is interesting.' \\ 'The case scenario was interesting.' \\ 'Learning at my convenience.' \\ 'Good overview if done at the start.' \\ 'Good for students not on the wards yet.' \\ 'It would be good at first CPBL to introduce things.' \\ Negative feedback
}

'Overcomplicated. A simple text conversation would be enough. No visual clones needed. Or maybe a picture series. However, the technology is not advanced enough to deal with what we need.'

'The chat functionality here is terrible but I know that making anything better takes exponentially more effort.'

'I would not recommend the use of this scenario in its current form. Needed to be done as a group with tutor.'

\section{Implications}

The use of Second Life as an education tool in psychiatric education does not seem effective in this form. It appears that the technology is not responsive enough; although it works with the more protocol-driven teaching for paramedics, it does not provide a realistic experience in psychiatry on the whole. We would therefore not recommend further attempts at developing psychiatric cases in Second Life. However, certain elements of this design were well received and it may be worth revisiting in the future, with the benefit of the lessons learnt from this experience (Box 3). We are planning to explore other interactive teaching methods in psychiatry such as interactive online PBL, which may be more suited to the subject matter.

Box 3 Lessons learnt from this experience

Training in the use of Second Life for students is required to allow the tool to be used to maximum benefit.

Second Life is best used in a tutor-led group setting.

Second Life could be useful as a educational resource designed to teach phenomenology to medical students and psychiatry trainees.

\section{Funding}

This project was exclusively funded by the Centre for Enterprise and Innovation at SGUL.

\section{Acknowledgements}

We would like to acknowledge the imput of Dr Vish Goel and Mr Kieran Walsh in the devleopment of the scenario.

\section{About the authors}

Jeremy Rampling is a specialty registrar in general adult psychiatry at the South West London \& St George's Mental Health NHS Trust. Aileen O'Brien is a senior lecturer in psychiatry at St George's, University of London. Keelyjo Hindhaugh is a specialty registrar in general adult psychiatry at the South West London \& St George's Mental Health NHS Trust. Luke Woodham is an e-learning technical developer in the Division of Population Health Sciences and Education, St George's, University of London. Sheetal Kavia is an e-learning projects manager in the Division of Population Health Sciences and Education, St George's, University of London.

\section{References}

1 Stott J. Medical education: learning the second way. BMJ 2007; 335 1122.

2 CNN Edge of Discovery. Can Second Life help Teach Doctors to Treat Patients? Cable News Network, 2009 (http://edition.cnn.com/2009/ TECH/03/30/doctors.second.life/cnnSTCText).

3 Wiecha J, Heyden R, Sternthal E, Merialdi M. Learning in a virtual world: experience with using second life for medical education. $J$ Med Internet Res 2010; 12: e1.

4 Reznek MA, Rawn CL, Krumel YM. Evaluation of the educational effectiveness of a virtual reality intravenous insertion simulator. Acad Emerg Med 2002; 9: 1319-25.

5 Joint Information Systems Committee. Problem-Based Learning in Virtual Interactive Worlds (PREVIEW) Final Report. JISC, 2008 (https:// curve.coventry.ac.uk/cu/items/38cd8bee-ae1d-d50c-e9c0a1dec639757a/1/PREVIEW\%20Final\%20Report.pdf).

6 Conradi E, Kavia S, Burden D, Rice A, Woodham L, Beaumont C, et al. Virtual patients in a virtual world: training paramedic students for practice. Med Teach 2009; 31: 713-20.

7 Toro-Troconis $M$, Mellström U, Partridge $M$, Meeran $K$, Barrett $M$, Higham J. Designing game-based learning activities for virtual patients in Second Life. J Cyber Rehabil 2008; 1: 225-38. 